\title{
Nanoscale
}

A Check for updates

Cite this: Nanoscale, 2019, 11, 3240

\section{High photoresponsivity and broadband photodetection with a band-engineered $\mathrm{WSe}_{2} / \mathrm{SnSe}_{2}$ heterostructure $\uparrow$}

\author{
Hui Xue, (D) *a Yunyun Dai, ${ }^{a}$ Wonjae Kim, ${ }^{b}$ Yadong Wang, ${ }^{a}$ Xueyin Bai, ${ }^{a}$ Mei Qi, ${ }^{a, c}$ \\ Kari Halonen, ${ }^{a}$ Harri Lipsanen ${ }^{a}$ and Zhipei Sun (iD) a,d
}

\begin{abstract}
van der Waals (vdW) heterostructures formed by stacking different two-dimensional layered materials have been demonstrated as a promising platform for next-generation photonic and optoelectronic devices due to their tailorable band-engineering properties. Here, we report a high photoresponsivity and broadband photodetector based on a $\mathrm{WSe}_{2} / \mathrm{SnSe}_{2}$ heterostructure. By properly biasing the heterostructure, its band structure changes from near-broken band alignment to type-III band alignment which enables high photoresponsivity from visible to telecommunication wavelengths. The highest photoresponsivity and detectivity at $532 \mathrm{~nm}$ are $\sim 588 \mathrm{~A} \mathrm{~W}^{-1}$ and $4.4 \times 10^{10}$ Jones and those at $1550 \mathrm{~nm}$ are $\sim 80 \mathrm{~A} \mathrm{~W}^{-1}$ and $1.4 \times 10^{10}$ Jones, which are superior to those of the current state-of-the-art layered transition metal dichalcogenides based photodetectors under similar measurement conditions. Our work not only provides a new method for designing high-performance broadband photodetectors but also enables a deep understanding of the band engineering technology in the vdW heterostructures possible for other applications, such as modulators and lasers.
\end{abstract}

Received 15th November 2018,

Accepted 21st January 2019

DOI: $10.1039 / c 8 n r 09248 f$

rsc.li/nanoscale material. For example, the bandgap of $\mathrm{MoS}_{2}$ is between $\sim 1.2$ $\mathrm{eV}$ (bulk) and $\sim 1.8 \mathrm{eV}$ (monolayer). ${ }^{19,20}$ Therefore, in principle, a $\mathrm{MoS}_{2}$ based photodetector typically cannot effectively detect light with a photon energy smaller than $1.2 \mathrm{eV}$. Such an intrinsic bandgap limitation poses a great challenge for various photonic applications (e.g., telecommunications).

van der Waals (vdW) heterojunctions, formed by stacking different individual 2D layered materials, can offer a new dimension in breaking the abovementioned intrinsic bandgap barrier. ${ }^{21-31}$ In general, three types of band alignments can be categorized when different 2D materials are stacked together, ${ }^{32}$ including type-I, ${ }^{33}$ type-II, ${ }^{34-38}$ and type-III ${ }^{39-43}$ band alignments. Thus far, most of the reported vdW heterostructure photodetectors are based on the type-II band alignment to utilize the excellent electron-hole pair separation ability in heterostructures. Recently, type-III band alignment has been explored as a platform for tunneling field effect transistors (TFETs) due to its enhanced tunneling current density. ${ }^{40,44-49}$ The 2D layered materials based type-III band alignment TFETs with improved responsivity and detectivity have been the focus of many recent studies in the visible wavelengths (e.g. 5.7 $\mathrm{A} \mathrm{W}^{-1}$ under $660 \mathrm{~nm},{ }^{50} 244 \mathrm{~A} \mathrm{~W}^{-1}$ under $550 \mathrm{~nm},{ }^{51} 180 \mathrm{~A} \mathrm{~W}^{-1}$ under $405 \mathrm{~nm}^{47}$ ). However, the applications of the type-III band alignment TFETs in the field of broadband photodetection have rarely been reported. In this paper, we report a high photoresponsivity and broadband photodetector based on a $\mathrm{WSe}_{2} / \mathrm{SnSe}_{2}$ heterostructure. By applying a large negative 
gate voltage and a reverse bias voltage, the heterojunction band structure changes from type-II to type-III band alignment and a band-to-band tunneling phenomenon occurs. The increased band bending at the heterojunction results in the tunneling-assisted transition which possibly allows photocurrent generation with energy smaller than the bandgap of $\mathrm{WSe}_{2}$ (e.g., $980 \mathrm{~nm}$ and $1550 \mathrm{~nm}$ ) for broadband photodetection (e.g. photo-excited electron tunnel from $\mathrm{WSe}_{2}$ to the conduction band of $\mathrm{SnSe}_{2}$ ). Under the type-III band alignment conditions, our heterojunction device shows a high photoresponsivity up to $\sim 588 \mathrm{~A} \mathrm{~W}^{-1}$ at a wavelength of $532 \mathrm{~nm}$ and $\sim 80 \mathrm{~A} \mathrm{~W}^{-1}$ at a wavelength of $1550 \mathrm{~nm}$. The corresponding photodetectivity reaches $4.4 \times 10^{10}$ Jones and $1.4 \times 10^{10}$ Jones for visible and telecommunication wavelengths, respectively.

\section{Results and discussion}

The optical image and schematic illustration of the $\mathrm{WSe}_{2} /$ $\mathrm{SnSe}_{2}$ heterostructure device are shown in Fig. 1a. Few-layer p-type $\mathrm{WSe}_{2}$ and n-type $\mathrm{SnSe}_{2}$ flakes, mechanically exfoliated from their bulk materials, are transferred onto a $280 \mathrm{~nm} \mathrm{SiO}_{2} /$ silicon substrate. An overlapping heterojunction region is formed by precisely controlling their locations. $\mathrm{Pd} / \mathrm{Au}$ metal contacts $(20 / 50 \mathrm{~nm})$ are patterned onto the $\mathrm{WSe}_{2}$ and $\mathrm{SnSe}_{2}$ flakes to collect holes and electrons. The thicknesses of the
$\mathrm{WSe}_{2}$ and $\mathrm{SnSe}_{2}$ few-layer flakes are determined by atomic force microscopy (AFM), giving the values of $6 \mathrm{~nm}$ and $15 \mathrm{~nm}$, respectively (ESI Fig. S1†). Raman spectroscopy is employed to confirm the high quality of our device after the fabrication processes (ESI Fig. S2†).

Before testing the electrical characteristics of the $\mathrm{WSe}_{2} /$ $\mathrm{SnSe}_{2}$ heterostructure, we also fabricated two individual $\mathrm{WSe}_{2}$ and $\mathrm{SnSe}_{2}$ based field effect transistors (FETs) to analyze the properties of $\mathrm{WSe}_{2}$ and $\mathrm{SnSe}_{2}$. WSe $\mathrm{WS}_{2}$ and $\mathrm{SnSe}_{2}$ FETs are fabricated on $\mathrm{Si} / \mathrm{SiO}_{2}$ substrates with $\mathrm{Pd} / \mathrm{Au}(20 / 50 \mathrm{~nm})$ as the drain and source electrodes. The transfer curves of the FETs (ESI Fig. S3 $\dagger$ ) show p-type characteristics for $\mathrm{WSe}_{2}$ and n-type characteristics for $\mathrm{SnSe}_{2}$, indicating that Pd can efficiently inject holes into $\mathrm{WSe}_{2}$ and electrons into $\mathrm{SnSe}_{2}$ when a negative gate voltage is applied. ${ }^{41,52}$ Moreover, the nonlinear output results of the $\mathrm{WSe}_{2}$ flake (ESI Fig. S3b $\dagger$ ) indicate the existence of a Schottky barrier at the contact region, while the $\mathrm{SnSe}_{2}$ results (ESI Fig. S3d $\dagger$ ) indicate that an ohmic contact is formed between $\mathrm{Pd}$ and $\mathrm{SnSe}_{2}$. Note that, since $\mathrm{SnSe}_{2}$ is a degenerated n-doped semiconductor, the band alignment of $\mathrm{SnSe}_{2}$ at the interface would be relatively unaffected by the $V_{\mathrm{g}}$ (see ESI Fig. S3c $\dagger$ ). ${ }^{35,43}$

Next, the electronic properties of the $\mathrm{WSe}_{2} / \mathrm{SnSe}_{2}$ heterojunction is studied. The device is characterized by applying a constant drain to source voltage $\left(V_{\mathrm{d}}\right)$ and a gate voltage $\left(V_{\mathrm{g}}\right)$ in the configuration shown in Fig. 1a. The device shows obvious p-type transport characteristics. The concentration and the (a)

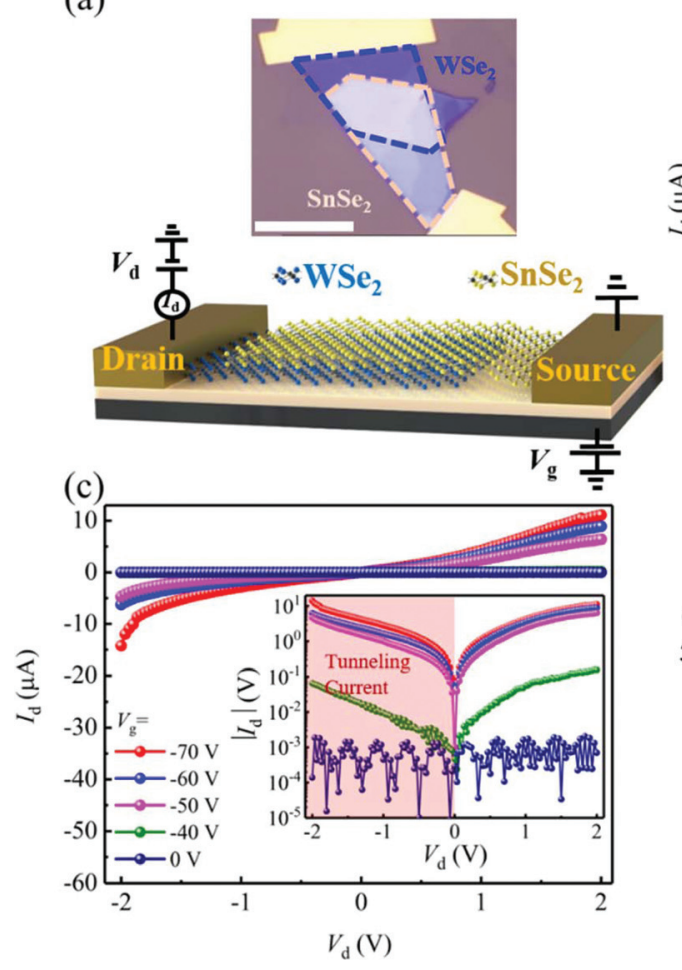

(b)

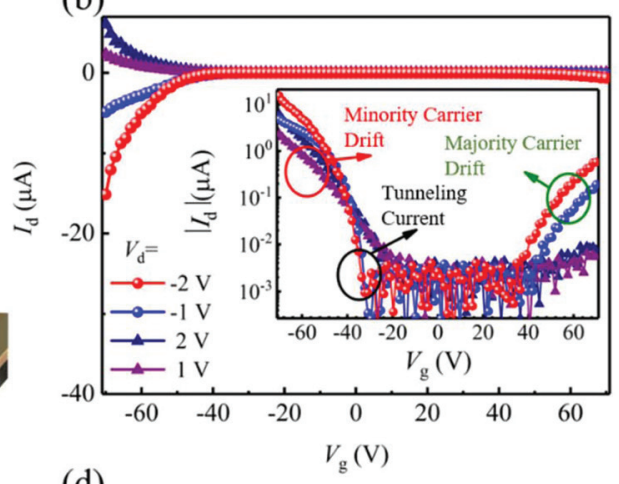

(d)

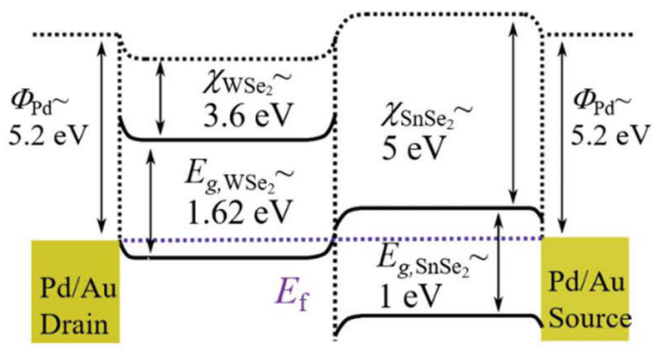

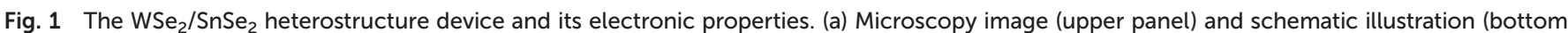

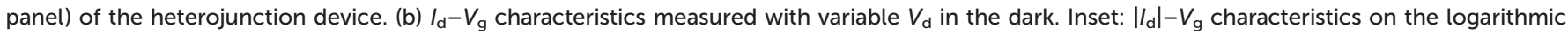

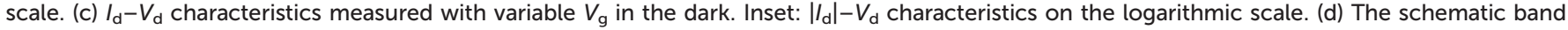
diagrams of the heterojunction. The scale bar is $10 \mu \mathrm{m}$. 
type of the carriers can be tuned by $V_{\mathrm{g}}$, at $V_{\mathrm{d}}=-2 \mathrm{~V}$; the $I_{\mathrm{d}}-V_{\mathrm{g}}$ result shows the gate-controlled current, with an on-off ratio of $\sim 10^{4}$ (see Fig. $1 \mathrm{~b}$ ). The reverse current $\left(I_{\text {reverse }}, V_{\mathrm{d}}<0 \mathrm{~V}\right)$ at $V_{\mathrm{g}}<-30 \mathrm{~V}$ is the band-to-band tunneling (BTBT) current, whose magnitude increases as the gate voltage decreases. The magnitude of $I_{\text {reverse }}$ at $V_{\mathrm{g}}>40 \mathrm{~V}$ increases as the gate voltage increases due to the majority carrier drifting. The forward current ( $I_{\text {forward }}, V_{\mathrm{d}}>0 \mathrm{~V}$ ) observed at $V_{\mathrm{g}}<-30 \mathrm{~V}$ can be attributed to the drifting of the minority carriers (see the discussion below). The magnitude of the reverse current increases with both positive and negative $V_{g}$ and the maximum of the reverse current is $15 \mu \mathrm{A}$ when $V_{\mathrm{d}}=-2 \mathrm{~V}$ and $V_{\mathrm{g}}=-70 \mathrm{~V}$. The gatedependent current-voltage $\left(I_{\mathrm{d}}-V_{\mathrm{d}}\right)$ characteristics are shown in Fig. 1c, where two important features can be observed. First, owing to the ultrathin thickness nature of the heterojunction, the magnitude of both $I_{\text {forward }}$ and $I_{\text {reverse }}$ can be significantly controlled by modifying the gate voltage. Second, since the breakdown voltage (i.e. $-0.01 \mathrm{~V}$ ) is very much less than $4 E_{\mathrm{g}} / q$, where $E_{\mathrm{g}}$ is the band gap of the semiconductors $(1.62 \mathrm{eV}$ of $\mathrm{WSe}_{2}$ and $1 \mathrm{eV}$ of $\mathrm{SnSe}_{2}$ ) and $q$ is the electron charge, the tunneling current (red region in the inset of Fig. 1c) can be attributed to Zener tunneling. ${ }^{53}$

The qualitative band alignment of the $\mathrm{WSe}_{2} / \mathrm{SnSe}_{2}$ heterostructure at zero bias is sketched in Fig. 1d based on the reported electron affinity values in the literature. ${ }^{54,55}$ Based on these values, a nearly broken band alignment is formed, where the Fermi level of $\mathrm{WSe}_{2}$ locates at the edge of its valence band and that of $\mathrm{SnSe}_{2}$ locates at the edge of its conduction band. The heterostructure resembles a $\mathrm{p}-\mathrm{n}$ diode with a staggered band gap offset. The bottom of the conduction band and the top of the valence band of $\mathrm{WSe}_{2}\left(\mathrm{SnSe}_{2}\right)$ are at $3.6 \mathrm{eV}(5.22 \mathrm{eV})$ and $5 \mathrm{eV}(6 \mathrm{eV})$, so that the offsets of the conduction band and valence band are about $1.4 \mathrm{eV}$ and $0.78 \mathrm{eV}$, respectively.

The transfer characteristics of the heterostructure can be understood through band diagrams (Fig. 2a-c). When there is no gate voltage (i.e. $\left.V_{g}=0 \mathrm{~V}\right)$, as shown in Fig. 2(a), the high Schottky barrier at the drain contact and the high difference of the conduction/valence band discontinuity restrain the current flow, leading to the zero dark current. Under these conditions, the device can be seen as a Schottky diode in series with a p-n junction and a resistor. When a negative gate voltage is applied (i.e. $V_{\mathrm{g}}<-30 \mathrm{~V}$ ) as shown in ESI Fig. S3b, $\dagger$ the doping level of $\mathrm{WSe}_{2}$ is increased and a near-ohmic contact is formed at the drain contact (the band bending direction at the drain contact shifts downwards). Thus, the model of the device under these conditions can be described as two resistors in series with a $\mathrm{p}^{+} \mathrm{n}$ junction. Therefore, when a reverse bias is applied (Fig. 2b), the valence band maximum of $\mathrm{WSe}_{2}$ shifts above the conduction band minimum of $\mathrm{SnSe}_{2}$, and the type of the band structure changes from near broken band alignment to broken band alignment (type-III). Thus, the electrons in the valence band of $\mathrm{WSe}_{2}$ can tunnel into the conduction band of the $\mathrm{SnSe}_{2}$ flake. This tunneling current can then be increased by increasing the reverse bias voltage, see Fig. 1c, due to the enlarged overlapping energy level between the valence band of $\mathrm{WSe}_{2}$ and the conduction band of $\mathrm{SnSe}_{2}$.
However, when a forward bias is applied (Fig. 2c), the band alignment changes from type-III to type-II, the barrier height at the heterojunction is reduced, and the majority carriers (electrons in $\mathrm{SnSe}_{2}$ and holes in $\mathrm{WSe}_{2}$ ) can overcome the interface barrier and drift to their counterparts, leading to a high forward current. The carrier transportation under the forward bias conditions follows the mechanism as described for the traditional $\mathrm{p}-\mathrm{n}$ diode, where the forward current increases exponentially with forward bias. ${ }^{40}$ To further analyze the transfer characteristics of the heterostructure, scanning photocurrent mapping at $\lambda=532 \mathrm{~nm}$ under a confocal optical microscope (objective $100 \times, \mathrm{NA}=0.75, P_{\text {laser }}=50 \mu \mathrm{W}$ (corresponding to a power intensity of $\left.8.5 \times 10^{3} \mathrm{~W} \mathrm{~cm}^{-2}\right)$ ) was performed, as shown in Fig. $2 d-\mathrm{f}$. The spot size $\left(\sim 0.6 \mu \mathrm{m}^{2}\right.$ at $\left.532 \mathrm{~nm}\right)$ of the incident laser is much smaller than the size $\left(\sim 50 \mu \mathrm{m}^{2}\right)$ of the heterojunction. When there is no bias (i.e. $V_{\mathrm{g}}=V_{\mathrm{d}}=0 \mathrm{~V}$ ), as mentioned above, the heterostructure resembles a $\mathrm{p}-\mathrm{n}$ junction. Thus, when there is an external light excitation, electronhole pairs will be generated and separated efficiently at the heterojunction (Fig. 2d) where the photo-generated electrons move to the $\mathrm{SnSe}_{2}$ flake while the holes move to the $\mathrm{WSe}_{2}$ flake, due to the built-in electric field. ${ }^{56}$ The part of the heterojunction near the drain contact shows a stronger photocurrent, which has also been observed in ref. 57 , and can be attributed to the lower sheet resistance of this region compared to those of other parts. Note that the photocurrent mapping results only indicate the region where the dominant photocurrent is generated. A small photocurrent (e.g., $\sim 1 \mathrm{nA}$ in Fig. 2d) can be observed in the drain part of the device. Under reverse bias with a negative $V_{\mathrm{g}}$, the $I_{\mathrm{d}}$ is governed by the BTBT, where carriers from the valence band of the $\mathrm{WSe}_{2}$ tunnel move to the conduction band of $\mathrm{SnSe}_{2}$, as mentioned above (Fig. 2b). Since the reversed $V_{\mathrm{d}}$ enhances the electric field in the heterojunction area, Fig. 2e exhibits a larger $I_{\mathrm{ph}}(\sim 50$ times larger than that in Fig. 2d) throughout the entire heterojunction. In contrast, while the device is at forward bias with a negative gate voltage, Pd can efficiently inject holes into $\mathrm{WSe}_{2}$, which can overcome the thermionic barrier and reach the source contact. In addition, the band bending at the heterojunction is released, leading to small $I_{\mathrm{ph}}$ generation in the heterojunction (Fig. 2f). Due to the fact that the direction of the built-in field at the drain part is the same as that of the external field, around $I_{\mathrm{ph}}=1 \mu \mathrm{A}$ is generated at the drain contact and the direction is towards the drain part.

The detailed photoresponse behavior of our device under a $\lambda=532 \mathrm{~nm}$ laser is also studied (Fig. 3). Note that, all the measurements are carried out by locating the laser spot at the heterojunction where the largest photocurrent is generated (Fig. 2e). The time-dependent $I_{\mathrm{ph}}$ under pulsed light illumination $(\lambda=532 \mathrm{~nm}, 10 \mu \mathrm{W})$ is firstly tested at $V_{\mathrm{g}}=0 \mathrm{~V}$ and $-70 \mathrm{~V}$ with different bias voltages, respectively, shown in Fig. 3a. The steep rise and fall edges indicate the fast response of the device. The device shows the photodiode-like behavior, where the $I_{\mathrm{ph}}$ increases significantly when it is at reverse bias compared with that of forward bias both at $V_{\mathrm{g}}=0 \mathrm{~V}$ and -70 $\mathrm{V}$. This can be explained by the fact that the increased band 
(a)

bending under the reverse bias conditions results in the efficient separation of the photo-generated electron-hole pairs, which coincides with our photocurrent mapping results (Fig. 2e and f). Since the forward bias results in a much lower $I_{\mathrm{ph}}$ compared with that from reverse bias, in the following discussion we will only focus on the $I_{\mathrm{ph}}$ under the condition of the reverse bias voltage.

Fig. $3 \mathrm{~b}$ and c illustrate the $I_{\mathrm{ph}}-V_{\mathrm{d}}$ curves of the heterostructure with fixed gate voltages $\left(V_{g}=0 \mathrm{~V}\right.$ and $V_{g}=-70 \mathrm{~V}$, respectively) under the $\lambda=532 \mathrm{~nm}$ laser with the laser power varying from $4 \mathrm{nW}$ to $37 \mu \mathrm{W}$. The magnitude of the $I_{\mathrm{ph}}$ strongly depends on the $V_{\mathrm{d}}$, due to the increased carrier drift velocity and the increased charge carrier separation efficiency, suggesting that a higher photocurrent can be readily achieved by applying a larger reversed $V_{\mathrm{d}}$. With the increase in the $P_{\text {laser }}$, the $I_{\mathrm{ph}}$ at reverse bias increases and approaches $8 \mu \mathrm{A}$ at $V_{\mathrm{d}}=$ $-3 \mathrm{~V}$ and $V_{g}=-70 \mathrm{~V}$ (Fig. 3c). Moreover, since the larger reversed $V_{\mathrm{d}}$ and $V_{\mathrm{g}}$ will lead to the larger electric field at the heterojunction region, the efficiency of the photo-excited carrier separation increases, resulting in a $\sim 62$-fold increase of

$I_{\mathrm{ph}}\left(P_{\text {laser }}=37 \mu \mathrm{W}, V_{\mathrm{d}}=-3 \mathrm{~V}\right)$ at $V_{\mathrm{g}}=-70 \mathrm{~V}$ compared with that (d)

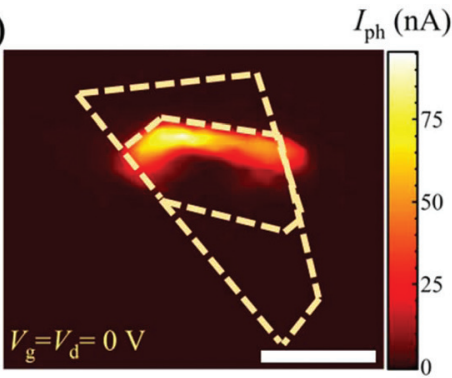

(e)

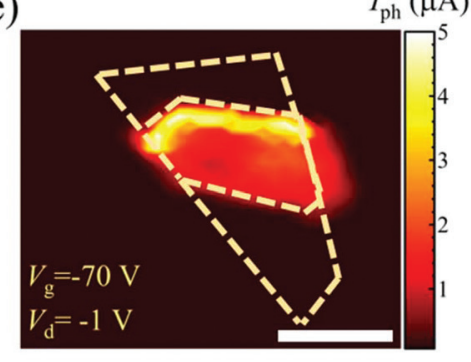

(f)

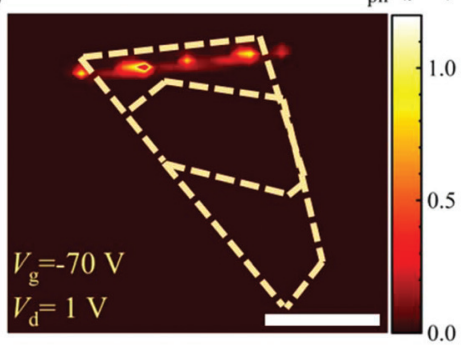

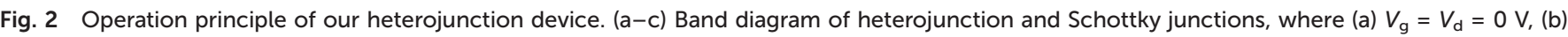
$V_{g}=-70 \mathrm{~V}, V_{d}=-1 \mathrm{~V}$ and (c) $V_{g}=-70 \mathrm{~V}, V_{d}=1 \mathrm{~V}$. The red/purple arrows indicate the carrier movement direction. The magenta arrows indicate the

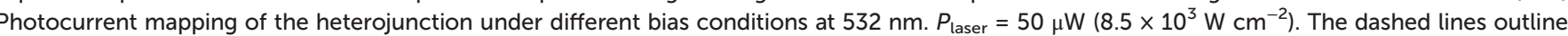
the flakes and heterojunction. The scale bar is $10 \mu \mathrm{m}$.

at $V_{\mathrm{g}}=0 \mathrm{~V}$. The $I_{\mathrm{ph}}$ as a function of $V_{\mathrm{g}}$ and $P_{\text {laser }}$ is plotted in Fig. 3d. Clearly, the $I_{\mathrm{ph}}$ increases with the decreasing of the gate voltage and a higher incident laser power. For example, when $P_{\text {laser }}=1 \mu \mathrm{W}$, the photocurrent is remarkable $(\sim 2 \mu \mathrm{A})$ under the condition of $V_{\mathrm{g}}<-40 \mathrm{~V}$, confirming that a smaller $V_{g}$ leads to a larger photocurrent.

To better understand the photoresponse properties of our device, several important figures of merit for photodetectors are studied, including the photoresponsivity $\left(R=I_{\mathrm{ph}} / P_{\text {laser }}\right)$, detectivity $\left(D=R \times s^{1 / 2} /\left(2 \times q \times I_{\text {dark }}\right)^{1 / 2}\right)$ and external quantum efficiency $\left(\mathrm{EQE}=\left(I_{\mathrm{ph}} / q\right) /\left(P_{\text {laser }} / h v\right)\right)$, where $q$ is the electron charge, $s$ is the illumination area, $I_{\text {dark }}$ is the current when the laser is off and $h v$ is the photon energy. ${ }^{17}$ The gate-dependent $R$ and EQE are shown in Fig. 3e. With a decrease in the $V_{g}$, the largest $R$ and EQE could reach $588 \mathrm{~A} \mathrm{~W}^{-1}$ and $1367 \%$, respectively, benefitting from the increased band bending which has been mentioned above. An EQE larger than 100\% has been previously observed, which is might due to the photo-excited carriers being recirculated many times before reaching the electrodes resulting from the long carrier lifetime..$^{50,58}$ The highest $D$ is estimated to be $4.4 \times 10^{10}$ Jones. The corresponding $R$ as a 
(a)
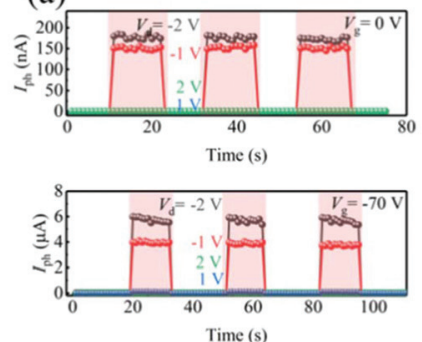

(c)
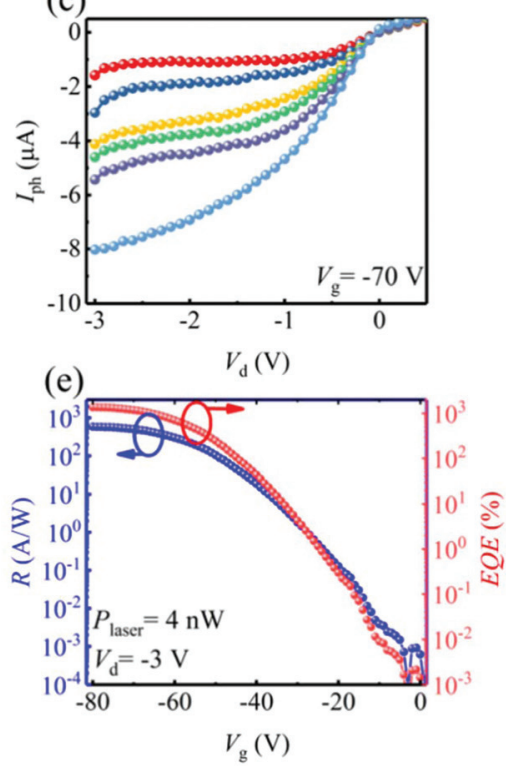

(b)
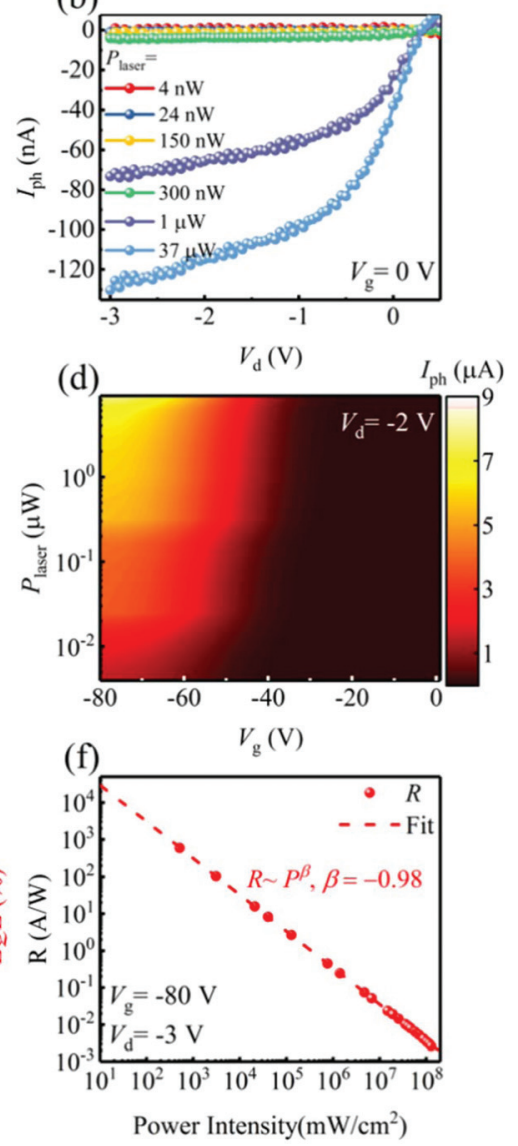

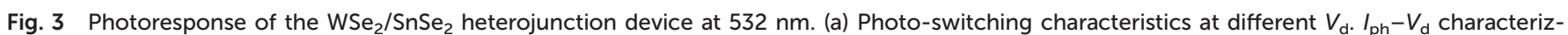

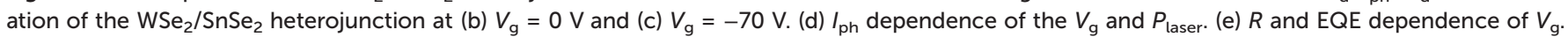
(f) $R$ measured with different input power intensities.

function of the incident power intensity is summarized in Fig. 3f. It is clearly observed that the $R$ linearly changes with the $P_{\text {laser }}$ in the $\log$ scale. By fitting the experimental data with the equation of $R \sim P^{\beta}$, the term $\beta$ is obtained at -0.98 for $532 \mathrm{~nm}$, indicating the superior photocurrent capability and excellent separation efficiency of photo-induced charge carriers.

Telecommunication wavelength detection is an important issue for various applications. Here, we test our heterojunction device at telecommunication wavelengths. The spatially resolved photocurrent mapping with a $\lambda=1550 \mathrm{~nm}$ laser $\left(V_{\mathrm{d}}=\right.$ $-1 \mathrm{~V}, V_{\mathrm{g}}=-70 \mathrm{~V}$ and $P_{\text {laser }}=10 \mu \mathrm{W}$ ) reveals pronounced photocurrent generation in the overlapped region (Fig. 4a). Fig. $4 \mathrm{~b}$ and c depict the gate-dependent photoresponse of the device under $980 \mathrm{~nm}$ and $1550 \mathrm{~nm}$ lasers, where $I_{\mathrm{ph}}$ shows the same tendency as that at $532 \mathrm{~nm}$. However, a threshold for the nearinfrared photon detection can be observed. For example, under the bias conditions, $V_{\mathrm{d}}=-2 \mathrm{~V}$ and $P_{\text {laser }}=10 \mu \mathrm{W}$, for $\lambda=$ $980 \mathrm{~nm}$, the photocurrent is not detectable when the $V_{\mathrm{g}}$ is larger than $-60 \mathrm{~V}$. As for the incident light at $\lambda=1550 \mathrm{~nm}$, under the same bias conditions, this threshold voltage shifts to $V_{\mathrm{g}}=-70 \mathrm{~V}$. This fascinating phenomenon can be attributed to the tunneling-assisted transition in the band-bending het- erojunction: an extremely negative gate voltage and a high reverse bias voltage increase the built-in electric field at the heterojunction, and the edges of the conduction band and valence band are tilted along the direction of the electric field. The electron and hole wave functions have a tail which extends into the band gap, enhancing the $\mathrm{e}-\mathrm{h}$ interactions with subbandgap photons. ${ }^{59}$ Thus, under such a high built-in electric field, our device has the potential to allow the photo-excited carrier generation with a photon energy smaller than the intrinsic band-gap of $\mathrm{WSe}_{2}$ and transfer to the conduction band of $\mathrm{SnSe}_{2} \cdot{ }^{53,60}$ The magnitude of the photocurrent under the $980 \mathrm{~nm}$ laser is around 2.6 times larger than that generated under the $1550 \mathrm{~nm}$ laser because the photon energy of $980 \mathrm{~nm}$ is higher than that of $1550 \mathrm{~nm}$. Note that there is no photoresponse of our device when it is forward biased even when the device is exposed to an intense light. Fig. $4 \mathrm{~d}$ and e show the $I_{\mathrm{ph}}-V_{\mathrm{d}}$ curves of the heterostructure with a fixed gate voltage $\left(V_{\mathrm{g}}=-70 \mathrm{~V}\right)$ under the $\lambda=980 \mathrm{~nm}$ and $1550 \mathrm{~nm}$ lasers with the laser power varying from $6 \mathrm{nW}$ to $400 \mu \mathrm{W}$ and $17 \mathrm{nW}$ to $400 \mu \mathrm{W}$, respectively. The magnitude of the $I_{\mathrm{ph}}$ shows strong dependence on the reverse bias voltage. This results from the larger built-in electric field at the larger reverse bias voltage. 
(a)

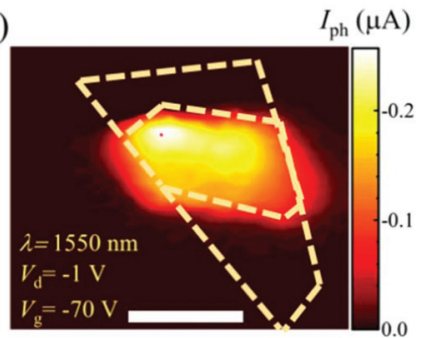

(c)

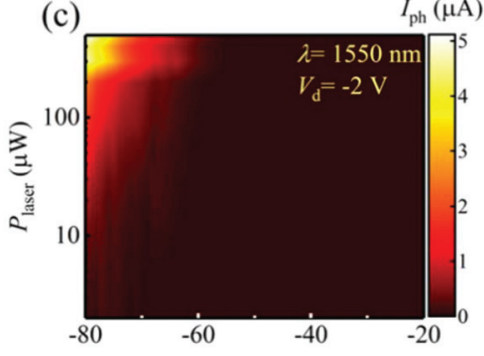

(e)

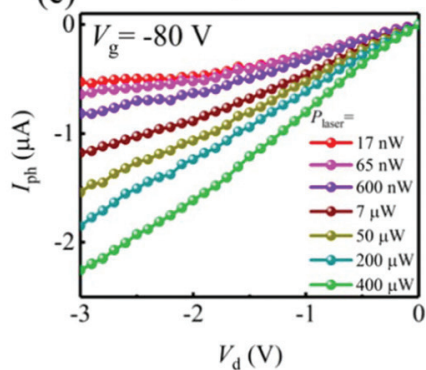

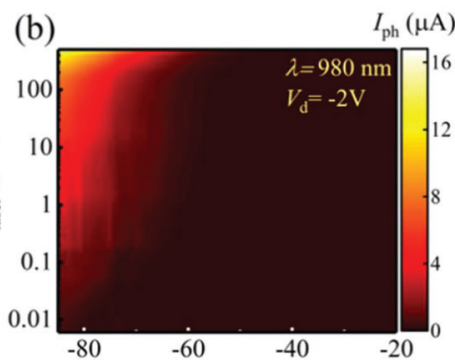

(d) $\quad V_{\mathrm{g}}(\mathrm{V})$
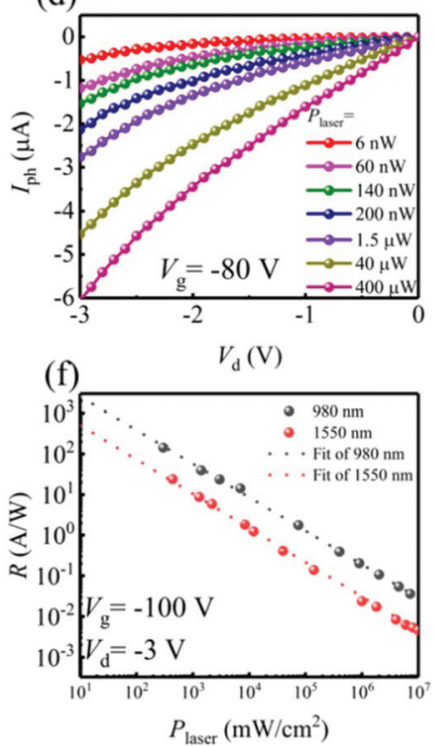

Fig. 4 Photoresponse of the WSe $\mathrm{S}_{2} / \mathrm{SnSe}_{2}$ heterojunction device at $980 \mathrm{~nm}$ and $1550 \mathrm{~nm}$. (a) Photocurrent mapping of the heterojunction under different bias conditions at $1550 \mathrm{~nm}$. $P_{\text {laser }}=10 \mu \mathrm{W}\left(2 \times 10^{5} \mathrm{~mW} \mathrm{~cm}{ }^{-2}\right) . I_{\mathrm{ph}}$ dependence of the $V_{\mathrm{g}}$ and $P_{\text {laser }}$ at (b) $980 \mathrm{~nm}$ and (c) $1550 \mathrm{~nm}$. $I_{\mathrm{ph}}-V_{\mathrm{d}}$ characterization of the $\mathrm{WSe}_{2} / \mathrm{SnSe}_{2}$ heterojunction at (d) $980 \mathrm{~nm}$ and (e) $1550 \mathrm{~nm}$. (f) $R$ at different input power intensities. The scale bar is $10 \mu \mathrm{m}$.

Fig. $4 \mathrm{f}$ depicts the $R$ of the device under $\lambda=980$ and $1550 \mathrm{~nm}$ as a function of the laser intensity. The maximum $R$ of our device (according to the fitting results) is $396 \mathrm{~A} \mathrm{~W}^{-1}$ at $980 \mathrm{~nm}$ $\left.\left(P_{\text {laser }}=100 \mathrm{~mW} \mathrm{~cm}\right)^{-2}\right)$ and is $80 \mathrm{~A} \mathrm{~W}^{-1}$ at $1550 \mathrm{~nm}\left(P_{\text {laser }}=\right.$ $100 \mathrm{~mW} \mathrm{~cm}{ }^{-2}$ ). The $R$ at the visible wavelength and telecommunication wavelength range is superior to those of the previously reported 2D materials and their heterostructure based photodetectors (see ESI Fig. S4 $\dagger$ ). The highest detectivity and EQE at these two wavelengths are $4.4 \times 10^{10}$ Jones, $500 \%$ and $1.4 \times 10^{10}$ Jones, $64 \%$, respectively. The time-dependent $I_{\mathrm{ph}}$ under pulsed light illumination (at $1550 \mathrm{~nm}$ ) is determined (ESI Fig. S5 $†$ ). The rise time and the decay time are 16 and $45 \mathrm{~ms}$, respectively. We believe that the speed of the device can be further improved by improving the crystal quality.

\section{Conclusions}

In summary, we report a high-sensitivity and broadband fewlayer $\mathrm{WSe}_{2} / \mathrm{SnSe}_{2}$ heterostructure based photodetector. The band-to-band tunneling mechanism allows the proposed device to detect light from visible to telecommunication wavelengths. The highest responsivity and detectivity at $532 \mathrm{~nm}$ are
$588 \mathrm{~A} \mathrm{~W}^{-1}$ and $4.4 \times 10^{10}$ Jones and those at $1550 \mathrm{~nm}$ are 80 $\mathrm{A} \mathrm{W}^{-1}$ and $1.4 \times 10^{10}$ Jones, which outperform the state-of-art 2D materials based photodetectors. Our work shows an attractive platform for practical applications of utilizing few-layer TMD based heterojunctions for high-performance optoelectronic devices (such as modulators ${ }^{61,62}$ and lasers). ${ }^{63-68}$

\section{Conflicts of interest}

There are no conflicts to declare.

\section{Acknowledgements}

We acknowledge funding from the Academy of Finland (Grant No. 276376, 284548, 295777, 304666, 312551, 314810, and 320167), the Academy of Finland Centre of Excellence program (project 312297), the Academy of Finland Flagship program (project 320167), Business Finland (OPEC, A-photonics), the European Union's Seventh Framework Program (Grant No. 631610), the European Union's Horizon 2020 research and innovation programme (Grant No. 820423, S2QUIP), Aalto 
Centre of Quantum Engineering, China Scholarship Council, and the provision of technical facilities of the Micronova, Nanofabrication Centre of Aalto University.

\section{References}

1 J. Wang, H. Fang, X. Wang, X. Chen, W. Lu and W. Hu, Small, 2017, 13, 1700894.

2 J. Liang, J. Zhang, Z. Li, H. Hong, J. Wang, Z. Zhang, X. Zhou, R. Qiao, J. Xu, P. Gao, Z. Liu, Z. Liu, Z. Sun, S. Meng, K. Liu and D. Yu, Nano Lett., 2017, 17, 75397543.

3 A. Saynatjoki, L. Karvonen, H. Rostami, A. Autere, S. Mehravar, A. Lombardo, R. A. Norwood, T. Hasan, N. Peyghambarian, H. Lipsanen, K. Kieu, A. C. Ferrari, M. Polini and Z. Sun, Nat. Commun., 2017, 8, 893.

4 H. Yang, H. Jussila, A. Autere, H. P. Komsa, G. J. Ye, X. H. Chen, T. Hasan and Z. P. Sun, ACS Photonics, 2017, 4, 3023-3030.

5 H. Hu, X. Yang, F. Zhai, D. Hu, R. Liu, K. Liu, Z. Sun and Q. Dai, Nat. Commun., 2016, 7, 12334.

6 Z. Q. Luo, D. D. Wu, B. Xu, H. Y. Xu, Z. P. Cai, J. Peng, J. Weng, S. Xu, C. H. Zhu, F. Q. Wang, Z. P. Sun and H. Zhang, Nanoscale, 2016, 8, 1066-1072.

7 A. Martinez and Z. P. Sun, Nat. Photonics, 2013, 7, 842-845.

8 A. Autere, C. R. Ryder, A. Saynatjoki, L. Karvonen, B. Amirsolaimani, R. A. Norwood, N. Peyghambarian, K. Kieu, H. Lipsanen, M. C. Hersam and Z. P. Sun, J. Phys. Chem. Lett., 2017, 8, 1343-1350.

9 P. Yan, H. Chen, J. Yin, Z. Xu, J. Li, Z. Jiang, W. Zhang, J. Wang, I. L. Li, Z. Sun and S. Ruan, Nanoscale, 2017, 9, 1871-1877.

10 H. Jussila, H. Yang, N. Granqvist and Z. P. Sun, Optica, 2016, 3, 151-158.

11 S. Das, R. Gulotty, A. V. Sumant and A. Roelofs, Nano Lett., 2014, 14, 2861-2866.

12 F. Bonaccorso, Z. Sun, T. Hasan and A. C. Ferrari, Nat. Photonics, 2010, 4, 611-622.

13 A. C. Ferrari, F. Bonaccorso, V. Fal'ko, K. S. Novoselov, S. Roche, P. Boggild, S. Borini, F. H. Koppens, V. Palermo, N. Pugno, J. A. Garrido, R. Sordan, A. Bianco, L. Ballerini, M. Prato, E. Lidorikis, J. Kivioja, C. Marinelli, T. Ryhanen, A. Morpurgo, J. N. Coleman, V. Nicolosi, L. Colombo, A. Fert, M. Garcia-Hernandez, A. Bachtold, G. F. Schneider, F. Guinea, C. Dekker, M. Barbone, Z. Sun, C. Galiotis, A. N. Grigorenko, G. Konstantatos, A. Kis, M. Katsnelson, L. Vandersypen, A. Loiseau, V. Morandi, D. Neumaier, E. Treossi, V. Pellegrini, M. Polini, A. Tredicucci, G. M. Williams, B. H. Hong, J. H. Ahn, J. M. Kim, H. Zirath, B. J. van Wees, H. van der Zant, L. Occhipinti, A. Di Matteo, I. A. Kinloch, T. Seyller, E. Quesnel, X. Feng, K. Teo, N. Rupesinghe, P. Hakonen, S. R. Neil, Q. Tannock, T. Lofwander and J. Kinaret, Nanoscale, 2015, 7, 4598-4810.

14 T. Juntunen, H. Jussila, M. Ruoho, S. H. Liu, G. H. Hu, T. Albrow-Owen, L. W. T. Ng, R. C. T. Howe, T. Hasan,
Z. P. Sun and I. Tittonen, Adv. Funct. Mater., 2018, 28, 1800480.

15 S. Li, Y. C. Lin, W. Zhao, J. Wu, Z. Wang, Z. Hu, Y. Shen, D. M. Tang, J. Wang, Q. Zhang, H. Zhu, L. Chu, W. Zhao, C. Liu, Z. Sun, T. Taniguchi, M. Osada, W. Chen, Q. H. Xu, A. T. S. Wee, K. Suenaga, F. Ding and G. Eda, Nat. Mater., 2018, 17, 535-542.

16 F. Bonaccorso, A. Lombardo, T. Hasan, Z. Sun, L. Colombo and A. C. Ferrari, Mater. Today, 2012, 15, 564-589.

17 Z. H. Sun and H. X. Chang, ACS Nano, 2014, 8, 4133-4156.

18 O. Lopez-Sanchez, D. Lembke, M. Kayci, A. Radenovic and A. Kis, Nat. Nanotechnol., 2013, 8, 497-501.

19 K. K. Kam and B. A. Parkinson, J. Phys. Chem. A, 1982, 86, 463-467.

20 K. F. Mak, C. Lee, J. Hone, J. Shan and T. F. Heinz, Phys. Rev. Lett., 2010, 105, 136805.

21 A. K. Geim and I. V. Grigorieva, Nature, 2013, 499, 419-425.

22 W. Zhang, Q. Wang, Y. Chen, Z. Wang and A. T. S. Wee, 2D Mater., 2016, 3, 022001.

23 H. Qiao, J. Yuan, Z. Q. Xu, C. Y. Chen, S. H. Lin, Y. S. Wang, J. C. Song, Y. Liu, Q. Khan, H. Y. Hoh, C. X. Pan, S. J. Li and Q. L. Bao, ACS Nano, 2015, 9, 1886-1894.

24 K. Zhang, T. Zhang, G. Cheng, T. Li, S. Wang, W. Wei, X. Zhou, W. Yu, Y. Sun, P. Wang, D. Zhang, C. Zeng, X. Wang, W. Hu, H. J. Fan, G. Shen, X. Chen, X. Duan, K. Chang and N. Dai, ACS Nano, 2016, 10, 3852-3858.

25 W. Kim, S. Arpiainen, H. Xue, M. Soikkeli, M. Qi, Z. Sun, H. Lipsanen, F. A. Chaves, D. Jimenez and M. Prunnila, ACS Appl. Nano Mater., 2018, 1, 3895-3902.

26 X. Yang, Z. Sun, T. Low, H. Hu, X. Guo, F. J. Garcia de Abajo, P. Avouris and Q. Dai, Adv. Mater., 2018, 30, e1704896.

27 H. Hu, X. Guo, D. Hu, Z. Sun, X. Yang and Q. Dai, Adv. Sci., 2018, 5, 1800175.

28 D. Hu, X. Yang, C. Li, R. Liu, Z. Yao, H. Hu, S. N. G. Corder, J. Chen, Z. Sun, M. Liu and Q. Dai, Nat. Commun., 2017, 8, 1471.

29 X. Yang, F. Zhai, H. Hu, D. Hu, R. Liu, S. Zhang, M. Sun, Z. Sun, J. Chen and Q. Dai, Adv. Mater., 2016, 28, 2931-2938.

30 H. Chen, J. Yin, J. Yang, X. Zhang, M. Liu, Z. Jiang, J. Wang, Z. Sun, T. Guo, W. Liu and P. Yan, Opt. Lett., 2017, 42, 4279-4282.

31 G. Hu, T. Albrow-Owen, X. Jin, A. Ali, Y. Hu, R. C. T. Howe, K. Shehzad, Z. Yang, X. Zhu, R. I. Woodward, T.-C. Wu, H. Jussila, J.-B. Wu, P. Peng, P.-H. Tan, Z. Sun, E. J. R. Kelleher, M. Zhang, Y. Xu and T. Hasan, Nat. Commun., 2017, 8, 278.

32 V. O. Ozcelik, J. G. Azadani, C. Yang, S. J. Koester and T. Low, Phys. Rev. B, 2016, 94, 035125.

33 J. W. Lee, Y. J. Choi, J. M. Yang, S. Ham, S. K. Jeon, J. Y. Lee, Y. H. Song, E. K. Ji, D. H. Yoon, S. Seo, H. Shin, G. S. Han, H. S. Jung, D. Kim and N. G. Park, ACS Nano, 2017, 11, 3311-3319.

34 D. Jariwala, S. L. Howell, K. S. Chen, J. Kang, V. K. Sangwan, S. A. Filippone, R. Turrisi, T. J. Marks, L. J. Lauhon and M. C. Hersam, Nano Lett., 2016, 16, 497-503. 
35 X. Zhou, N. Zhou, C. Li, H. Song, Q. Zhang, X. Hu, L. Gan, H. Li, J. Lü, J. Luo, J. Xiong and T. Zhai, 2D Mater., 2017, 4, 025048.

36 L. Ye, H. Li, Z. Chen and J. Xu, ACS Photonics, 2016, 3, 692-699.

37 M. Buscema, J. O. Island, D. J. Groenendijk, S. I. Blanter, G. A. Steele, H. S. van der Zant and A. Castellanos-Gomez, Chem. Soc. Rev., 2015, 44, 3691-3718.

38 H. Xue, Y. Wang, Y. Dai, W. Kim, H. Jussila, M. Qi, J. Susoma, Z. Ren, Q. Dai, J. Zhao, K. Halonen, H. Lipsanen, X. Wang, X. Gan and Z. Sun, Adv. Funct. Mater., 2018, 1804388.

39 K. E. Aretouli, D. Tsoutsou, P. Tsipas, J. Marquez-Velasco, S. A. Giamini, N. Kelaidis, V. Psycharis and A. Dimoulas, ACS Appl. Mater. Interfaces, 2016, 8, 23222-23229.

40 X. Liu, D. Qu, H. M. Li, I. Moon, F. Ahmed, C. Kim, M. Lee, Y. Choi, J. H. Cho, J. C. Hone and W. J. Yoo, ACS Nano, 2017, 11, 9143-9150.

41 T. Roy, M. Tosun, M. Hettick, G. H. Ahn, C. Hu and A. Javey, Appl. Phys. Lett., 2016, 108, 083111.

42 C. Li, X. Yan, X. Song, W. Bao, S. Ding, D. W. Zhang and P. Zhou, Nanotechnology, 2017, 28, 415201.

43 X. Yan, C. Liu, C. Li, W. Bao, S. Ding, D. W. Zhang and P. Zhou, Small, 2017, 13, 1701478.

44 M. Li, D. Esseni, J. J. Nahas, D. Jena and H. G. Xing, IEEE J. Electron Devices Soc., 2015, 3, 206-213.

45 A. Nourbakhsh, A. Zubair, M. S. Dresselhaus and T. Palacios, Nano Lett., 2016, 16, 1359-1366.

46 D. Tan, X. Wang, W. Zhang, H. E. Lim, K. Shinokita, Y. Miyauchi, M. Maruyama, S. Okada and K. Matsuda, Small, 2018, 14, e1704559.

47 Q. A. Vu, J. H. Lee, V. L. Nguyen, Y. S. Shin, S. C. Lim, K. Lee, J. Heo, S. Park, K. Kim, Y. H. Lee and W. J. Yu, Nano Lett., 2017, 17, 453-459.

48 R. Yan, S. Fathipour, Y. Han, B. Song, S. Xiao, M. Li, N. Ma, V. Protasenko, D. A. Muller, D. Jena and H. G. Xing, Nano Lett., 2015, 15, 5791-5798.

49 K. Murali and K. Majumdar, IEEE J. Electron Devices Soc., 2018, 65, 4141-4148.

50 C. Lan, C. Li, S. Wang, T. He, T. Jiao, D. Wei, W. Jing, L. Li and Y. Liu, ACS Appl. Mater. Interfaces, 2016, 8, 18375-18382.

51 X. Zhou, X. Hu, S. Zhou, H. Song, Q. Zhang, L. Pi, L. Li, H. Li, J. Lu and T. Zhai, Adv. Mater., 2018, 30, 1703286.
52 W. Zhang, M.-H. Chiu, C.-H. Chen, W. Chen, L.-J. Li and A. T. S. Wee, ACS Nano, 2014, 8, 8653-8661.

53 J. I. Pankove, Optical processes in semiconductors, PrenticeHall, Englewood Cliffs, N.J, 1971.

54 X. Duan, C. Wang, A. Pan, R. Yu and X. Duan, Chem. Soc. Rev., 2015, 44, 8859-8876.

55 Y. Guo and J. Robertson, Appl. Phys. Lett., 2016, 108, 233104.

56 M. Buscema, D. J. Groenendijk, G. A. Steele, H. S. van der Zant and A. Castellanos-Gomez, Nat. Nanotechnol., 2014, 5, 4651.

57 Y. Deng, Z. Luo, N. J. Conrad, H. Liu, Y. Gong, S. Najmaei, P. M. Ajayan, J. Lou, X. Xu and P. D. Ye, ACS Nano, 2014, 8, 8292-8299.

58 G. Konstantatos, M. Badioli, L. Gaudreau, J. Osmond, M. Bernechea, F. P. Garcia de Arquer, F. Gatti and F. H. Koppens, Nat. Nanotechnol., 2012, 7, 363-368.

59 J. B. You, H. Kwon, J. Kim, H. H. Park and K. Yu, Opt. Express, 2017, 25, 4284-4297.

60 Y. Zhou, Y. H. Liu, J. Cheng and Y. H. Lo, Nano Lett., 2012, 12, 5929-5935.

61 Z. Sun, A. Martinez and F. Wang, Nat. Photonics, 2016, 10, 227-238.

62 A. Autere, H. Jussila, A. Marini, J. R. M. Saavedra, Y. Y. Dai, A. Saynatjoki, L. Karvonen, H. Yang, B. Amirsolaimani, R. A. Norwood, N. Peyghambarian, H. Lipsanen, K. Kieu, F. J. G. de Abajo and Z. P. Sun, Phys. Rev. B, 2018, 98, 115426.

63 Z. Sun, T. Hasan and A. C. Ferrari, Phys. E, 2012, 44, 10821091.

64 Z. P. Sun, T. Hasan, F. Torrisi, D. Popa, G. Privitera, F. Q. Wang, F. Bonaccorso, D. M. Basko and A. C. Ferrari, ACS Nano, 2010, 4, 803-810.

65 F. Bonaccorso and Z. P. Sun, Opt. Mater. Express, 2014, 4, 63-78.

66 J. Bogusławski, Y. Wang, H. Xue, X. Yang, D. Mao, X. Gan, Z. Ren, J. Zhao, Q. Dai, G. Soboń, J. Sotor and Z. Sun, Adv. Funct. Mater., 2018, 28, 1801539.

67 A. Autere, H. Jussila, Y. Dai, Y. Wang, H. Lipsanen and Z. Sun, Adv. Mater., 2018, 30, 1705963.

68 D. A. Li, H. Jussila, L. Karvonen, G. J. Ye, H. Lipsanen, X. H. Chen and Z. P. Sun, Sci. Rep., 2015, 5, 15899. 